\title{
Cooperative gating between ion channels
}

\author{
Kee-Hyun Choi ${ }^{1,2}$ \\ ${ }^{1}$ Chemical Kinomics Research Center, Future Convergence Research Division, Korea Institute of Science and Technology \\ (KIST), Seoul, Republic of Korea \\ 2 Biological Chemistry Program, University of Science and Technology (UST), Daejeon, Republic of Korea
}

\begin{abstract}
Cooperative gating between ion channels, i.e. the gating of one channel directly coupled to the gating of neighboring channels, has been observed in diverse channel types at the single-channel level. Positively coupled gating could enhance channel-mediated signaling while negative coupling may effectively reduce channel gating noise. Indeed, the physiological significance of cooperative channel gating in signal transduction has been recognized in several in vivo studies. Moreover, coupled gating of ion channels was reported to be associated with some human disease states.

In this review, physiological roles for channel cooperativity and channel clustering observed in vitro and in vivo are introduced, and stimulation-induced channel clustering and direct channel cross linking are suggested as the physical mechanisms of channel assembly. Along with physical clustering, several molecular mechanisms proposed as the molecular basis for functional coupling of neighboring channels are covered: permeant ions as a channel coupling mediator, concerted channel activation through the membrane, and allosteric mechanisms. Also, single-channel analysis methods for cooperative gating such as the binomial analysis, the variance analysis, the conditional dwell time density analysis, and the maximum likelihood fitting analysis are reviewed and discussed.
\end{abstract}

Key words: Ion channel - Cooperative gating - Functional coupling - Single-channel analysis

\section{Introduction}

Channel cooperativity has been observed in patch-clamp studies of diverse types of ion channels (Fig. 1). Positively coupled gating has been reported in many types of ligandgated channels: nicotinic acetylcholine receptors (nAChRs) (Schindler et al. 1984; Yeramian et al. 1986; Keleshian et al. 2000), AMPA (a-amino-3-hydroxyl-5-methyl-4-isoxazolepropionate) receptors (Vaithianathan et al. 2005; Suppiramaniam et al. 2006), ryanodine receptors (RyRs) (Marx et al. 1998, 2001; Laver et al. 2004), 1,4-dihydropyridine receptors (DHPRs) (Hymel et al. 1988), $\mathrm{P}_{2} \mathrm{X}_{2}$ receptors (Ding and Sachs 2002), and hyperpolarization-activated cyclic nucleotide-gated (HCN) channels (Dekker and Yellen 2006). In addition, L-type $\mathrm{Ca}^{2+}$ channels (Navedo et al. 2010), bacterial

Correspondence to: Kee-Hyun Choi, Chemical Kinomics Research Center, Future Convergence Research Division, Korea Institute of Science and Technology (KIST), Hwarangno 14-Gil 5, Seongbuk-Gu, Seoul 136-791, Republic of Korea

E-mail: keehyun@kist.re.kr voltage-gated $\mathrm{K}^{+}$channels (KcsA) (Molina et al. 2006), and gap junction channels (Chen and DeHaan 1992; Manivannan et al. 1992; Veenstra et al. 1994) are other examples of positively coupled gating. In contrast to positive coupling, negative coupling has been rarely observed in electrophysiological studies. Negative cooperativity was observed in toxin-modified $\mathrm{Na}^{+}$channels (Iwasa et al. 1986) and $\mathrm{Na}^{+}$ channels in nerve fibers (Neumcke and Stampfli 1983).

\section{Physiological roles of cooperativity}

\section{Positive cooperativity}

Positively coupled gating, in which one channel is more likely to be open when the other channel is open than when it is closed, could serve as a strategy to enhance channel-mediated signaling in response to cellular signals. In particular, positive cooperativity of AMPA and NMDA ( $N$-methyl-Daspartate) receptors in the hippocampus has been proposed as the underlying mechanism for long-term potentiation, 
a cellular mechanism of synaptic strengthening for learning and memory (Malenka 2003a, 2003b). Cooperativity between $\mathrm{N}$-type $\mathrm{Ca}^{2+}$ channels in the presynaptic terminal was shown to regulate synaptic vesicle fusion, thereby controlling neurotransmitter release at the neuromuscular junction (Shahrezaei et al. 2006; Matveev et al. 2009).

Cooperative gating of RyRs in rat cardiac myocytes altered dynamic patterns of intracellular $\mathrm{Ca}^{2+}$ such as $\mathrm{Ca}^{2+}$ spark duration, amplitude, and frequency, consequently influencing $\mathrm{Ca}^{2+}$ signaling (McCall et al. 1996; Xiao et al. 1997). Positively coupled gating between RyRs in cardiac muscle was proposed as the mechanism for termination of $\mathrm{Ca}^{2+}$ release (Marx et al. 2001). In this mechanism, synchronous channel closing reduces stochastic channel reactivation that otherwise would occur due to $\mathrm{Ca}^{2+}$ passing through neighboring channels. Interestingly, positively coupled gating of L-type $\mathrm{Ca}^{2+}$ channels in cardiac and arterial myocytes was suggested to be associated with cardiac diseases such as hypertension and arrhythmias (Navedo et al. 2010). Coupled gating of L-type $\mathrm{Ca}^{2+}$ channels was enhanced in hypertensive smooth muscle, and mutant L-type $\mathrm{Ca}^{2+}$ channels that cause arrhythmias exhibited increased gating cooperativity.

Cooperativity of voltage-gated $\mathrm{Na}^{+}$channels was suggested to account for a rapid initiation of action potentials observed in cortical neurons (Naundorf et al. 2006). This abrupt initial rising phase was not described by the Hodgkin-Huxley model that assumes independent channel gating (Hille 2001), but described by positive cooperativity among $\mathrm{Na}^{+}$channels. Effects of positive coupling between $\mathrm{Na}^{+}$ channels on action potentials were confirmed by computational modeling as well as in vitro experiments where the action potential initiation observed in vivo was reproduced by reducing the density of available $\mathrm{Na}^{+}$channels by applying a $\mathrm{Na}^{+}$channel specific toxin. However, alternative mechanisms are not excluded; an abrupt initiation of the action potential was well-described also by the modified Hodgkin-Huxley model that includes variability in subthreshold potentials but not cooperativity between $\mathrm{Na}^{+}$ channels (McCormick et al. 2007).

\section{Negative cooperativity}

Negative coupling, i.e. an open state of one channel disfavors opening of the other channel, may effectively reduce channel gating noise produced by stochastic transitions between channel open and closed states. Random fluctuations in the number of open channels have been shown to be a dominant electrical noise source in excitable cells, affecting the membrane potential (Schneidman et al. 1998; Diba et al. 2004; Jacobson et al. 2005). Effects of gating noise from voltagegated $\mathrm{Na}^{+}$and $\mathrm{K}^{+}$channels on action potentials have been extensively studied theoretically and experimentally (Chow and White 1996; Schneidman et al. 1998; White et al. 1998; Kretzberg et al. 2001; van Rossum et al. 2003; Diba et al. 2004; Jacobson et al. 2005). Membrane potential fluctuations generated by stochastic channel gating limit the reliability of neuronal response to stimuli by altering dynamics in neurons such as firing frequency and timing (Chow and White 1996; Schneidman et al. 1998; White et al. 1998; Kretzberg et al. 2001; van Rossum et al. 2003).

Stochastic gating of hyperpolarization-activated cation $\left(\mathrm{I}_{\mathrm{h}}\right)$ channels in pyramidal neurons was shown to contribute to fluctuations in membrane potential, thus damaging action potential fidelity; a 100-fold decrease in the ability to detect a signal and the temporal precision of firing (Kole et al. 2006). The small single-channel conductance of $\mathrm{I}_{\mathrm{h}}$ channels $(\sim 0.7$ pS) may play a role in reducing stochastic gating noise for reliable neuronal functions. However, minimizing gating noise through negative cooperativity between neighboring channels would be a more general and efficient way for reliable signal transduction in noisy cellular environments.

\section{Channel clustering}

Co-localization of channels is prerequisite for functional channel coupling (Fig. 2). Indeed, channel clustering has been observed in most of ion channels exhibiting cooperative gating. Channel clustering is often associated with alteration in channel activity and/or channel kinetics. However, the physical mechanisms of channel assembly mostly remain unclear.

\section{Channel assembly in vitro}

To detect physically assembled channels, analytical ultracentrifugation sedimentation velocity studies and native SDS-PAGE/Western blots are used. Species with higher retention on native SDS-PAGE gels and higher sedimentation coefficients than the functional channel unit are expected when channels are clustered. These biochemical assays were carried out with purified KcsA channels, revealing the existence of KcsA supra-molecular assemblies (Molina et al. 2006). Physically associated pairs of RyRs from skeletal and cardiac muscle were also observed in sucrose gradient centrifugation and immune-blotting experiments (Marx et al. 1998, 2001). As another example, Torpedo nAChRs were purified as a mixture of monomers and dimers, which was evident in sucrose gradient experiments (Schindler et al. 1984). However, in vitro biochemical assays are limited to channel proteins whose purification is possible in large quantity and whose physical interaction is strong enough even under detergent-solubilized conditions. Also, if other endogenous cellular molecules are involved in channel clustering, channel assembly will not be detected with purified channel proteins alone. 
A Independent channels
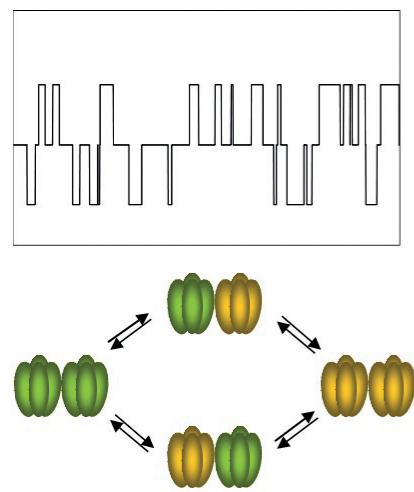

B Positive coupling
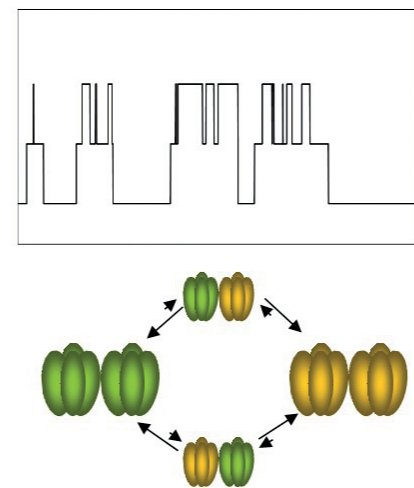

C Negative coupling

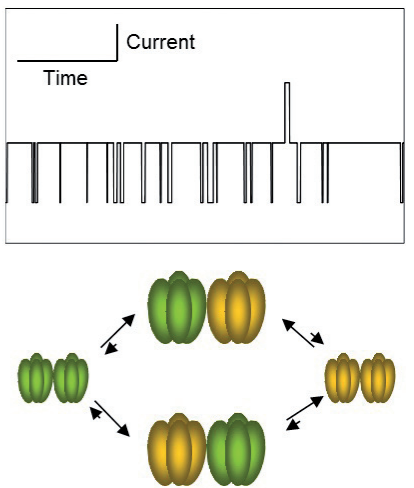

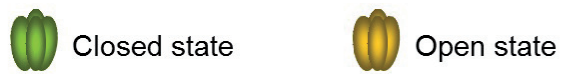

Figure 1. Electrophysiological evidence for cooperative gating. Using single-channel recordings, coupled gating can be detected at the single-molecule level. Channels in green are in their closed states and yellow channels are in their open states. A. For independent channels, the state of one channel does not affect gating of neighboring channels. Two channels exhibit independent gating in a simulated single-channel record. B. Positively coupled channels undergo synchronous channel opening and closing as shown in a simulated singlechannel record of two positively cooperative channels. C. Negative coupling between adjacent channels leads to a heterogeneous channel population (a mixture of open and closed channels at most of time). A single-channel record simulated for two negatively coupled channels shows lower fluctuations in the number of open channels than independent channels shown in A. All simulations were performed using QuB software (www.qub.buffalo.edu) (Qin et al. 2000).

\section{Sub-cellular channel localization}

Localization of HCN4 channels in caveolae, a membrane sub-domain, was detected in rabbit sinoatrial cells (Barbuti et al. 2004). Western blotting of isolated caveolae provided evidence of HCN4 channel localization. Furthermore, chemical disruption of caveolae impaired HCN4 localization, affecting HCN4 channel kinetics. Freeze/fracture electron microscopy studies revealed that gap junction channels aggregate into clusters (Goodenough 1976; Kensler et al. 1979). RyR clusters in skeletal and cardiac muscle have been also observed in situ by electron microscopy (Block et al. 1988; Saito et al. 1988;

\section{A Sub-cellular localization}

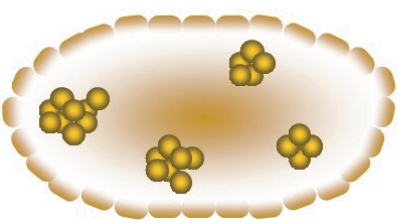

B Stimulation-induced clustering

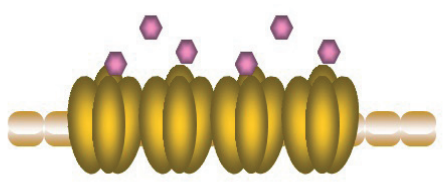

\section{Direct channel cross linking}

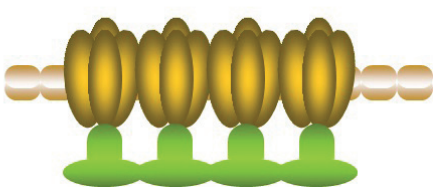

Figure 2. Channel clustering. For functional coupling between adjacent channels, transient and/or permanent co-localization of channels is prerequisite. A. Sub-cellular localization is frequently observed in cooperative channels. Clustered channels in the cell surface are presented as yellow balls. B. Channel assembly is often induced by cellular stimuli. For example, some ligand-gated ion channels are clustered transiently and spontaneously upon ligand (pink hexagons) binding. C. Direct and physical channel-channel interactions are another way to cluster channels. Some ion channels are found to physically interact with neighboring channels through their C-terminal or N-terminal tails. In some cases, scaffold proteins mediate and/or assist channel assembly. Cytoskeletal or extracellular proteins directly cross link channels through intracellular domains or extracellular parts of channels, respectively. Ion channels (yellow) are linked by intracellular scaffold proteins (green). 
Flucher and Franzini-Armstrong 1996; Franzini-Armstrong et al. 1999). In addition, electron microscopic (Geng et al. 2003) and pharmacological (Hehl and Neumcke 1993) data on ATP-sensitive $\mathrm{K}^{+}$channels suggested their clustering.

\section{Stimulation-induced channel clustering}

Dimerization of Torpedo nAChRs in the postsynaptic membrane is transiently induced during synaptic stimulation (Dunant et al. 1989). Changes in the population of monomeric and dimeric nAChRs by electrical stimuli were observed in situ using rapid freeze-fracture techniques. Intact electric organ tissues from Torpedo were rapidly frozen in the absence and presence of electrical stimuli, fractured, and then observed using electron microscopy. Monomeric nAChRs (globular form, $9 \mathrm{~nm}$ in diameter) were a primary form found in the freeze-fracture replicas of un-stimulated membranes. Following a single nerve impulse, however, nAChR dimers (elongated form, $18 \mathrm{~nm}$ in diameter) were momentarily formed. The decrease in the number of monomers was twice the increase in the number of dimers. The time course of abrupt change in nAChR ultra-structures was comparable with that of the fast transient postsynaptic current evoked by electric signals, implying that channel assembly might be associated with channel activation.

The inositol 1,4,5-triphosphate receptor $\left(\mathrm{IP}_{3} \mathrm{R}\right)$, a ligandgated $\mathrm{Ca}^{2+}$ channel is another example of stimulation-induced channel clustering (Tateishi et al. 2005). $\mathrm{IP}_{3} \mathrm{Rs}$ in the endoplasmic reticulum (ER) membrane release $\mathrm{Ca}^{2+}$ from ER to cytosol upon $\mathrm{IP}_{3}$ binding. Green fluorescence proteinfused $\mathrm{IP}_{3}$ Rs were assembled into clusters following application of $\mathrm{IP}_{3}$-generating agents, which implies that $\mathrm{IP}_{3}$-stimulation induces a channel conformational change to the open state, thereby initiating channel clustering. This hypothesis was further tested using $\mathrm{IP}_{3} \mathrm{R}$ mutants that impair either $\mathrm{IP}_{3}$ binding or channel activity without affecting ligand binding ability. Both $\mathrm{IP}_{3} \mathrm{R}$ mutants failed to form clusters, supporting the hypothesis that ligand-induced conformational changes are required for channel clustering.

\section{Direct channel cross linking}

The physical linkage between ion channels has been observed in several systems where coupled gating was found: RyRs (Marx et al. 1998, 2001), AMPA receptors (Suppiramaniam et al. 2006), L-type $\mathrm{Ca}^{2+}$ channels (Navedo et al. 2010), and cystic fibrosis transmembrane conductance regulators (CFTRs) (Wang et al. 2000). Purified RyRs reconstituted in lipid bilayers exhibited coupled gating, which required FK506 binding proteins, FKBP12 and FKBP12.6 for skeleton and cardiac muscle type RyRs, respectively (Marx et al. 1998, 2001). AMPA receptors isolated from rat brain showed positive cooperativity induced by dextran sulfate, a mimic of sulfated polysaccharides in synapses (Suppiramaniam et al. 2006). Heparin, a synaptic polysaccharide, modulates AMPA receptor activity by linking them physically (Hall et al. 1996). Addition of dextran sulfate promoted synchronous openings and closings of AMPA receptors, whose positive coupling was confirmed by a binomial analysis. In addition, coupled gating of L-type $\mathrm{Ca}^{2+}$ channels was found to be associated with spontaneous and transient interactions between neighboring channels through their C-termini (Navedo et al. 2010). AKAP150, a kinase anchoring protein appeared to enhance L-type $\mathrm{Ca}^{2+}$ channel cooperativity, presumably by facilitating the physical interaction between the C-terminal tails of channels.

Lastly, interactions between CFTRs were facilitated by CAP70, a multivalent CFTR binding protein (Wang et al. 2000). CAP70 bound to the C-termini of CFTRs linked them through its PDZ domains, stimulating CFTR activity. Correlated physical and functional coupling between CFTRs was further tested using a bivalent monoclonal antibody that specifically recognizes the $\mathrm{C}$-terminal residues of CFTR. Two CFTRs linked by a bivalent antibody exhibited increased CFTR activity. Current amplitude histograms of CFTRs showed a positive deviation from a binomial distribution upon antibody addition, indicating that positive coupling was induced by linking neighboring channels. However, it is not clear whether CFTR activation induced by CAP70 is resulted from coupled gating because no test for channel cooperativity was carried out. $\mathrm{Na}^{+} / \mathrm{H}^{+}$exchanger regulatory factor (NHE-RF) also mediates CFTR interactions by cross linking the CFTR C-termini, increasing CFTR activity (Raghuram et al. 2001). In this case, however, CFTRs did not show cooperative gating based on a binomial analysis even though multiple CFTRs were simultaneously activated by NHE-RF.

\section{Molecular basis of channel coupling}

Although functional coupling is often observed with physical clustering for some ion channels (Marx et al. 1998, 2001; Wang et al. 2000; Suppiramaniam et al. 2006), a physical linkage between channels does not necessarily affect channel gating. Additional steps are required to link channel clustering to functional coupling that affects channel gating. Several molecular mechanisms have been proposed to account for coupled gating of multiple channels (Fig. 3).

\section{Permeant ions as a channel coupling mediator}

One possible mechanism is that channels sense local concentration of their permeant ions (Fig. 3A). Indeed, clustered DHPRs are regulated by $\mathrm{Ca}^{2+}$, its permeant ion (Imredy 
and Yue 1992). Inactivation of DHPRs by prior $\mathrm{Ca}^{2+}$ influx was thought to be an intrinsic negative feedback that does not require cooperative channel interactions. However, the DHPR inactivation rate observed in two-channel patches from guinea pig myocytes was faster than that in one-channel patches while two-channel patches with one active and one inactive channel showed a reduced inactivation rate. These results indicate that $\mathrm{Ca}^{2+}$ influx from both channels are required for inhibitory channel coupling rather than physical association of adjacent channels. When BAPTA (1,2-bis-(2aminophenoxy)ethane- $N, N, N^{\prime}, N^{\prime}$-tetraacetic acid), a $\mathrm{Ca}^{2+}$ chelator was applied into the cytosol, the inactivation rate in two-channel patches slowed down more than that in onechannel patches, suggesting that $\mathrm{Ca}^{2+}$ mediates interactions among DHPRs. The two cytosolic $\mathrm{Ca}^{2+}$ inhibition sites were proposed: one near the pore and the other more distant from it. The site near the pore mediates $\mathrm{Ca}^{2+}$-sensitive inactivation by $\mathrm{Ca}^{2+}$ flow through its own pore, accounting for channel inactivation even when bulk cytosolic $\mathrm{Ca}^{2+}$ is chelated. In contrast, the site distant from the pore is involved in coupled inhibition through local $\mathrm{Ca}^{2+}$ influx within overlapping domains of neighboring channels, which can be diminished by $\mathrm{Ca}^{2+}$ chelators. Therefore, inhibitory coupling between DHPRs arises from shared and localized $\mathrm{Ca}^{2+}$ diffusion from adjacent channels.

\section{Concerted channel activation through the membrane}

Interactions mediated by the membrane in which channels are embedded provide another possible physical mechanism for coupled gating (Fig. 3B). In case of mechano-sensitive (MS) channels, channel activities are regulated by relative movements of the channel and/or the membrane at the channel-membrane interface (Martinac 2004; Sukharev and Corey 2004; Kung 2005; Ursell et al. 2007). Conformational changes of MS channels can be induced by membrane deformation (the bilayer model) or deformation of cytoskeletal or extracellular proteins directly connected to channels (the tethered model). In any case, channel displacement with respect to the membrane could produce hydrophobic mismatch between the lipid bilayer and the channel, triggering channel conformational changes. To the extent that channel conformation changes produce local changes in bilayer thickness, mechanosensitivity in channel gating will also lead to coupled gating.

The bilayer model where membrane movement (stretching or bending) promotes conformational changes of ion channels through changes in membrane geometry is the dominant mechanism for cooperative gating of prokaryotic MS channels (Martinac 2004). Purified and reconstituted bacterial MS channels still exhibit mechano-sensitivity, indicating that they can directly sense membrane deformation. Activation of E. coli MS channels was observed when lysophospholipids or amphipaths were inserted into the membrane, perturbing the local membrane curvature (Martinac et al. 1990; Perozo et al. 2002) although it cannot be ruled out that lysophospholipids and/or amphipaths activate MS channels by direct interactions with channels rather than through perturbation of the membrane curvature.

In the tether mechanism, MS channels are directly linked to cytoskeletal or extracellular proteins that can transmit force to the channels and thus activate them (Sukharev and Corey 2004). The tether model was proposed to describe MS channels in eukaryotic cells with an extensive cytoskeletal network adjacent to the membrane. Direct evidence for the tether model was observed in two types of transient receptor potential (TRP) channels: zebrafish TRPN1 and Drosophila TRPA1 channels (Corey et al. 2004; Siemens et al. 2004; Sollner et al. 2004). Cadherin 23 protein and the $\mathrm{N}$-terminal ankyrin domain of TRPA1 were identified as a tether for TRPN1 and TRPA1 channels, respectively. Cadherin 23 links TRPN1 to the extracellular matrix while the ankyrin domain of TRPA1 is attached to the cytoskeleton. Cytoskeletal/extracellular matrix deformation could displace the channels with respect to the lipid bilayer.

Mechano-sensitivity has been also observed in other types of channels including voltage-gated $\mathrm{Na}^{+}$channels (Lundbaek et al. 2004), L-type and $\mathrm{N}$-type $\mathrm{Ca}^{2+}$ channels (Calabrese et al. 2002; Lyford et al. 2002), Shaker-IR $\mathrm{K}^{+}$channels $(\mathrm{Gu}$ et al. 2001), large conductance $\mathrm{Ca}^{2+}$-activated $\mathrm{K}^{+}$channels (Tang et al. 2003), ATP-sensitive $\mathrm{K}^{+}$channels (Van Wagoner 1993), cardiac muscarinic $\mathrm{K}^{+}$channels (Pleumsamran and Kim 1995), and NMDA receptors (Paoletti and Ascher 1994). The observation of mechano-sensitivity in diverse types of ion channels that are not classified as MS channels suggests that they will be also sensitive to perturbations in the membrane including conformational changes of other membrane proteins. Further studies will be necessary to determine whether mechano-sensitivity actually causes coupled gating in these systems.

\section{Allosteric mechanism}

Neighboring channels might act as an allosteric modulator. The conformational state of one channel in clusters can influence conformational changes in neighboring channels, possibly by changing binding affinity of ligand in case of ligand-gated channels (Fig. 3C). Although direct visualization of allosteric conformational changes is limited by spatial and temporal resolution of current techniques, indirect evidence such as a Hill coefficient can be obtained. Bacterial chemoreceptors are the best-studied model system for allostery in membrane proteins (Sourjik 2004; Parkinson et al. 2005; Kentner and Sourjik 2006). Allosteric interactions between neighboring chemo-receptors were proposed to account for remarkable sensitivity of bacterial cells to their chemo-effectors. The signal amplification in E. coli cells was quantified 
A Permeant ions

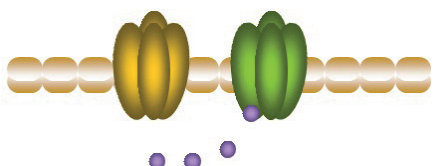

B Through the membrane

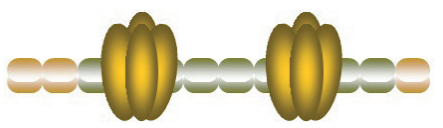

C Allosteric mechanism

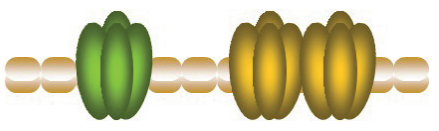

Figure 3. Molecular basis of functional coupling of neighboring channels. Once channels are physically clustered, the next step is necessary for coupled gating. A. Permeant ions can act as a channel coupling mediator. Some $\mathrm{Ca}^{2+}$ channels gate cooperatively through their permeant $\mathrm{Ca}^{2+}$ ions. $\mathrm{Ca}^{2+}$ ions (purple balls) passing into the cell through open channels (yellow) bind to the intracellular $\mathrm{Ca}^{2+}$ binding sites of adjacent channels, inhibiting neighboring channel activity. $\mathrm{Ca}^{2+}$ bound channels are in their closed states (green). B. Ion channels can undergo concerted conformational changes through the membrane. Conformational changes of one channel could induce changes in membrane tension. The distorted membrane (grey) consequently triggers conformational changes of neighboring channels. C. Linked channels can act as a positive or negative allosteric modulator of other channels in cluster. When acting as a positive modulator, one open channel will favor the opening of its adjacent channels. Two positively coupled channels are shown in their open states (yellow).

A Binomial analysis

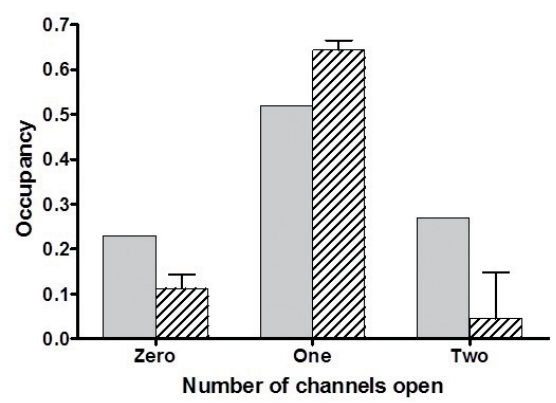

C Conditional dwell time density analysis

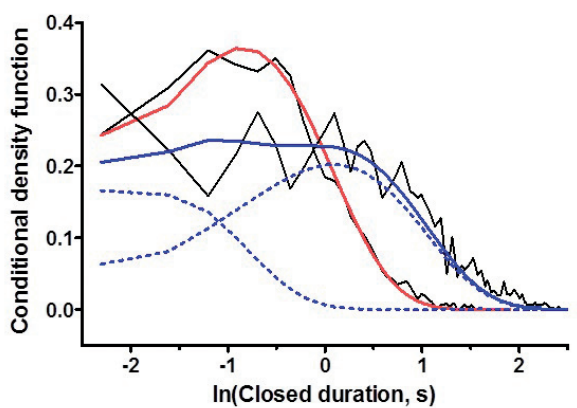

B Variance analysis

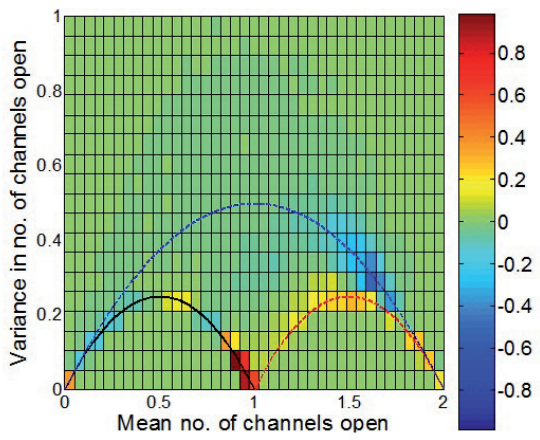

D Maximum likelihood fitting analysis

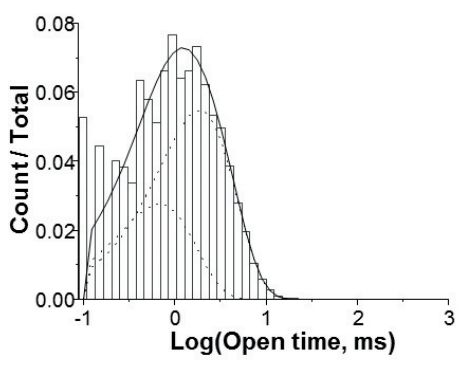

Figure 4. Single-channel analysis methods for cooperative gating. Channel cooperativity can be detected using single-channel recordings (Choi and Licht 2012). A. A binomial analysis is a commonly utilized method due to its explicit nature. Positive or negative deviations from binomial predictions indicate positive or negative coupling, respectively. A bar graph shows a negative deviation (dashed bars) from a binomial distribution (grey bars) observed in two-channel patches of ATP-sensitive $\mathrm{K}^{+}$channels, suggesting negative cooperativity. This graph was adapted from (Choi and Licht 2012). B. A variance analysis is another binomial theorem-based method using a steady-state property (open probability) of channels. A blue dotted line is theoretically calculated for two identical channels, a black solid line is for one channel, and a red dashed line is for two negatively coupled channels. Experimentally observed ATP-sensitive $\mathrm{K}^{+}$channel gating in two-channel patches follows the theoretical value for negative cooperativity as shown in pseudo-colored squares. This graph was generated from the records used in (Choi and Licht 2012), but unpublished before. C. A conditional dwell time density analysis utilizes kinetic information of channel gating. Conditional open or closed time densities are obtained when the other channel is open or closed throughout. Conditional closed time density functions of ATP-sensitive $\mathrm{K}^{+}$channels in two-channel patches show a longer mean closed time when the other channel is open (red line) than that when the other channel is closed (blue line), indicating negative cooperativity. This graph was adapted from (Choi and Licht 2012). D. A maximum likelihood fitting analysis also utilizes channel kinetic information. Single-channel data are fitted using the two kinetic models: one for independent channels and the other for coupled channels. A maximum likelihood ratio obtained from the maximum likelihood estimation algorithm tells which model fits the data better. For open times of ATP-sensitive $\mathrm{K}^{+}$channels, the independent and coupled gating models show the same log likelihood. The open time distribution fitted to a double exponential function (solid line with two dotted lines) using the cooperative model is shown. This graph was generated from the records used in (Choi and Licht 2012), but unpublished before. 
using a fluorescence resonance energy transfer assay in vivo, providing evidence for allosteric interactions among chemoreceptors (Sourjik and Berg 2002, 2004). Chemo-receptors amplified chemotactic signals (changes in chemo-effector concentrations) 35-fold in a highly cooperative fashion (a Hill coefficient of $\sim 10$ ), well-described by the classical Monod-Wyman-Changeux model. Allosteric channel interactions in clusters could thus be one of the mechanisms for coupled gating of clustered channels.

\section{Single-channel analysis methods for cooperative gating}

\section{Binomial analysis}

Once synchronous open and closed transitions are observed, which is highly suggestive of cooperativity, a binomial analysis on current amplitude histograms (distribution of the current levels) is the appropriate first step to test gating cooperativity (Fig. 4A). The observed distributions of current amplitudes are compared with those predicted for independent channels, i.e. binomial distributions. Deviations from a binomial distribution are indicative of cooperative gating; positive deviations (more simultaneous openings than expected from independent gating) are consistent with positive coupling while negative deviations are consistent with negative cooperativity.

Positive deviations from a binomial distribution have been observed in $\mathrm{P}_{2} \mathrm{X}_{2}$ receptors (Ding and Sachs 2002), native RyRs from skeletal and cardiac muscle (Marx et al. 1998, 2001), RyRs reconstituted in lipid bilayers (Laver et al. 2004), purified DHPRs in lipid bilayers (Hymel et al. 1988), reconstituted AMPA receptors from rat brain (Vaithianathan et al. 2005; Suppiramaniam et al. 2006), nAChRs from chick myocytes (Keleshian et al. 2000), Torpedo nAChRs reconstituted in planar membranes (Schindler et al. 1984), native gap junction channels (Chen and DeHaan 1992; Manivannan et al. 1992), recombinant connexin 45 gap junction channels (Veenstra et al. 1994), and purified KcsA in giant liposomes (Molina et al. 2006). Negative deviations have been found in toxin-modified $\mathrm{Na}^{+}$channels in neuroblastoma cells (Iwasa et al. 1986).

Three assumptions must be made to interpret a binomial analysis for cooperative interactions: the channel record is stationary (no global changes in channel activity within a given record length), the number of channels is correctly estimated, and the channels are identical (Kenyon and Bauer 2000). The necessity of these assumptions often leads to complication in interpreting results from a binomial analysis. First, reliable estimate of the number of channels in patch is not always straightforward. It is likely the case when the number of channels is estimated by the maximum number of simultaneous openings with a low open probability. In this case, the number of channels is likely to be underestimated, resulting in overestimation of positive deviations from a binomial distribution. In addition, independent but non-identical channels (independent channels with different open probabilities) show apparent negative deviations from a binomial distribution, and are not distinguishable from negatively coupled channels. Thus, a binomial analysis is often suggestive but not conclusive in case of both positive and negative cooperativity.

\section{Variance analysis}

A variance analysis is another way to detect cooperative gating (Traynelis and Jaramillo 1998). A mean-variance plot can be generated by calculating the variance of the macroscopic current as a function of the mean current (Fig. 4B). The unitary current of one channel (singlechannel current) can be estimated from an initial slope of the mean-variance plot based on a binomial theorem (Traynelis and Jaramillo 1998). If channels gate independently, the unitary current calculated from a mean-variance plot will be the same as the single-channel current. However, if channels are positively coupled, synchronous open and closed events will generate higher fluctuations in the mean macroscopic current than that expected from independent channels. For negatively coupled channels, synchronous opening and closing transitions will be suppressed, generating lower fluctuations. Thus, the estimated unitary current from coupled channels will deviate from the single-channel current; positive cooperativity will increase the estimated unitary current whereas negative cooperativity will decrease it (Liu and Dilger 1993). HCN2 channels showed the larger unitary current than the singlechannel current, suggesting positive cooperativity (Dekker and Yellen 2006). Negative coupling between $\mathrm{Na}^{+}$channels in nerve fibers was also suggested by a variance analysis (Neumcke and Stampfli 1983).

However, a variance analysis has the same complication as a binomial analysis because it is also a binomial theorem-based method. First, if channels do not have identical open probabilities, the observed variance will be less than that predicted for identical channels (Nedelman and Wallenius 1986). A similar problem arises if channels are non-stationary and varying on a timescale slower than a record length because they will also appear non-identical. Finally, incorrect estimate of the number of channels will lead to incorrect prediction of the variance since the variance is linearly proportional to the number of channels (Sakmann and Neher 1995). Incorrect estimate of the number of channels can be further exaggerated by lack of stationarity. Thus, stationarity of the channel records must be tested to distinguish stationary data from non-stationary data (Choi and Licht 2012). 
Moreover, the direct comparison of the single-channel current to the unitary current derived from a mean-variance plot should be done with cautions because two different recording environments (single-channel recording versus whole-cell recording) will result in differences in bandwidth and noise sources, leading to variability in currents. Comparison of the variance calculated from multiple channels to that from one channel in the same patch would be helpful to see any effects from the different recording environments. Simulation of multiple-channel data and one-channel data is another way to test reliability of this analysis.

Overall, a steady-state (equilibrium) method like binomial and variance analyses does not use kinetic information of channel gating. Collective behaviors of coupled channels can be revealed by a kinetic analysis, which could provide evidence for or against channel cooperativity. Therefore, a further analysis with channel gating kinetics is often necessary to confirm coupled gating.

\section{Conditional dwell time density analysis}

Several methods to analyze gating kinetics to detect channel cooperativity share a common basis (Iwasa et al. 1986; Keleshian et al. 1994; Blunck et al. 1998; Keleshian et al. 2000; Ding and Sachs 2002; Laver et al. 2004): determining whether the dwell time density of one channel is conditional on the other channel open or closed (Fig. 4C) (Yeo et al. 1989). Independent channels will have identical conditional dwell time densities. For coupled channels, however, two conditional dwell time densities will not be the same. When two interacting channels are present, closed and/or open time densities of one channel will depend on whether the other channel is open or closed. If channels are positively coupled, the opening rate will increase and/or the closing rate will decrease when neighboring channels are open. For negatively coupled channels, neighboring open channels will decrease the opening rate and/or increase the closing rate. Similarly, the channel opening and/or closing rates can be influenced by the closed state of neighboring channels.

$\mathrm{nAChR}$ records showed that open time densities are identical whether a neighboring channel is open or closed (Keleshian et al. 2000). However, the mean closed time of one channel is longer when the other channel is closed than when it is open, suggesting that positive interactions affect channel opening but not closing. Similar results were also observed in RyRs (Laver et al. 2004). The opening rates were faster in the presence of open channels, suggesting that channel opening depends on other open channels (positive coupling). Cooperative gating of $\mathrm{P} 2 \mathrm{X}_{2}$ receptors was studied also using a kinetic analysis (Ding and Sachs 2002). The mean open time of single-channel patches is shorter than that of multiple-channel patches, suggesting that the closing rate is influenced by positive coupling. A conditional dwell time density analysis was also used to detect negative interactions between two toxin-modified $\mathrm{Na}^{+}$channels (Iwasa et al. 1986). However, the opening rates could not be determined due to the multiple closed states, and the closing rates were identical, failing to detect negative cooperativity.

In general, coupled gating can be detected by alteration in conditional open/closed time densities. However, a conditional dwell time analysis is not easy to implement. If more than two channels are present, more combinations of conditional dwell time densities will be generated, making it more complicated. A finite length of records also limits the power of this analysis because the number of dwells may not be sufficient to generate statistically reliable dwell time densities. This problem becomes more serious when multiple channels with a low open probability are present because there will be a small number of multiple events. Finally, this method compares the mean open and closed times, i.e. the mean closing and opening rates. Since more than one type of open/closed transitions are likely to be present in the real gating mechanisms, if specific opening or closing transitions influenced by coupled gating are buried in all-point dwell time densities, they might not be detectable.

\section{Maximum likelihood fitting analysis}

Coupled gating can be also detected by a maximum likelihood fitting analysis with the independent and cooperative models (Fig. 4D) (Ding and Sachs 2002). When channels are independent, the channel opening and closing rates are not affected by whether the neighboring channels are open or closed. In a two-independent-channel system, the opening rate from the two-channel closed state to the onechannel open state $\left(k_{\mathrm{CO} 1}\right)$ is simply twice as fast as that from the one-channel open state to the two-channel open state $\left(k_{\mathrm{O} 1 \mathrm{O} 2}\right)\left(k_{\mathrm{CO} 1}=2 k_{\mathrm{O} 1 \mathrm{O} 2}\right)$. The closing rate from the twochannel open state to the one-channel open state $\left(k_{\mathrm{O} 2 \mathrm{O} 1}\right)$ is also twice as fast as that from the one-channel open state to the two-channel closed state $\left(k_{\mathrm{O} 1 \mathrm{C}}\right)\left(k_{\mathrm{O} 2 \mathrm{O} 1}=2 k_{\mathrm{O} 1 \mathrm{C}}\right)$. Single-channel data can be fitted with rate constraints $\left(k_{\mathrm{CO} 1}=2 k_{\mathrm{O} 1 \mathrm{O} 2}, k_{\mathrm{O} 2 \mathrm{O} 1}=2 k_{\mathrm{O} 1 \mathrm{C}}\right.$; independent model $)$ or without them (cooperative model). Using a maximum likelihood ratio test, a kinetic model that describes experimental data better can be determined. This analysis was applied to single-channel records of $\mathrm{P}_{2} \mathrm{X}_{2}$ receptors (Ding and Sachs 2002). The cooperative model fitted $P 2 X_{2}$ data better than the independent model, suggesting channel cooperativity. However, a maximum likelihood fitting analysis is modeldependent. Thus, it could suffer from ambiguous data fitting when channel gating has the complex underlying kinetic mechanisms. 


\section{Concluding remarks}

Coupled gating has been observed in diverse ion channel types at the single-channel level. Although cooperative gating could possibly play a physiologically important role in various systems, it is challenging to investigate in vivo effects of channel coupling because various proteins are involved in regulation of channel activity in cells. Thus, the molecular mechanisms of cooperative channel gating are still mostly unknown. In addition, existing single-channel analysis methods to detect coupled gating are limited, necessitating the development of more explicit and unambiguous methods.

Acknowledgements. I thank Dr. Stuart Licht for comments on the manuscript. This work was funded by the KIST Institutional Programs (Grant No. 2Z03942, 2E24090, and 2V03209) from Korea Institute of Science and Technology, and by the Creative Fusion Research Program (Grant No. CAP-12-1) from Korea Research Council of Fundamental Science and Technology.

\section{References}

Barbuti A., Gravante B., Riolfo M., Milanesi R., Terragni B., DiFrancesco D. (2004): Localization of pacemaker channels in lipid rafts regulates channel kinetics. Circ. Res. 94, 1325-1331 http://dx.doi.org/10.1161/01.RES.0000127621.54132.AE

Block B. A., Imagawa T., Campbell K. P., Franzini-Armstrong C. (1988): Structural evidence for direct interaction between the molecular components of the transverse tubule/sarcoplasmic reticulum junction in skeletal muscle. J. Cell. Biol. 107, $2587-2600$ http://dx.doi.org/10.1083/jcb.107.6.2587

Blunck R., Kirst U., Riessner T., Hansen U. (1998): How powerful is the dwell-time analysis of multichannel records? J. Membr. Biol. 165, 19-35 http://dx.doi.org/10.1007/s002329900417

Calabrese B., Tabarean I. V., Juranka P., Morris C. E. (2002): Mechanosensitivity of N-type calcium channel currents. Biophys. J. 83, 2560-2574 http://dx.doi.org/10.1016/S0006-3495(02)75267-3

Chen Y. H., DeHaan R. L. (1992): Multiple-channel conductance states and voltage regulation of embryonic chick cardiac gap junctions. J. Membr. Biol. 127, 95-111 http://dx.doi.org/10.1007/BF00233282

Choi K. H., Licht S. (2012): ATP-sensitive potassium channels exhibit variance in the number of open channels below the limit predicted for identical and independent gating. PLoS One 7, e37399 http://dx.doi.org/10.1371/journal.pone.0037399

Chow C. C., White J. A. (1996): Spontaneous action potentials due to channel fluctuations. Biophys. J. 71, 3013-3021 http://dx.doi.org/10.1016/S0006-3495(96)79494-8

Corey D. P., Garcia-Anoveros J., Holt J. R., Kwan K. Y., Lin S. Y., Vollrath M. A., Amalfitano A., Cheung E. L., Derfler B. H.,
Duggan A., Geleoc G. S., Gray P. A., Hoffman M. P., Rehm H. L., Tamasauskas D., Zhang D. S. (2004): TRPA1 is a candidate for the mechanosensitive transduction channel of vertebrate hair cells. Nature 432, 723-730 http://dx.doi.org/10.1038/nature03066

Dekker J. P., Yellen G. (2006): Cooperative gating between single HCN pacemaker channels. J. Gen. Physiol. 128, 561-567 http://dx.doi.org/10.1085/jgp.200609599

Diba K., Lester H. A., Koch C. (2004): Intrinsic noise in cultured hippocampal neurons: experiment and modeling. J. Neurosci. 24, 9723-9733 http://dx.doi.org/10.1523/JNEUROSCI.1721-04.2004

Ding S., Sachs F. (2002): Evidence for non-independent gating of $\mathrm{P} 2 \mathrm{X} 2$ receptors expressed in Xenopus oocytes. BMC Neurosci. 3, 17 http://dx.doi.org/10.1186/1471-2202-3-17

Dunant Y., Garcia-Segura L. M., Muller D., Parducz A. (1989): Momentary alteration of the postsynaptic membrane during transmission of a single nerve impulse. Proc. Natl. Acad. Sci. USA 86, 1717-1720 http://dx.doi.org/10.1073/pnas.86.5.1717

Flucher B. E., Franzini-Armstrong C. (1996): Formation of junctions involved in excitation-contraction coupling in skeletal and cardiac muscle. Proc. Natl. Acad. Sci. USA 93, 8101-8106 http://dx.doi.org/10.1073/pnas.93.15.8101

Franzini-Armstrong C., Protasi F., Ramesh V. (1999): Shape, size, and distribution of $\mathrm{Ca}(2+)$ release units and couplons in skeletal and cardiac muscles. Biophys. J. 77, 1528-1539 http://dx.doi.org/10.1016/S0006-3495(99)77000-1

Geng X. H., Li L. H., Watkins S., Robbins P. D., Drain P. (2003): The insulin secretory granule is the major site of KATP channels of the endocrine pancreas. Diabetes 52, 767-776 http://dx.doi.org/10.2337/diabetes.52.3.767

Goodenough D. A. (1976): The structure and permeability of isolated hepatocyte gap junctions. Cold Spring Harb. Symp. Quant. Biol. 40, 37-43

http://dx.doi.org/10.1101/SQB.1976.040.01.006

Gu C. X., Juranka P. F., Morris C. E. (2001): Stretch-activation and stretch-inactivation of Shaker-IR, a voltage-gated K+ channel. Biophys. J. 80, 2678-2693 http://dx.doi.org/10.1016/S0006-3495(01)76237-6

Hall R. A., Vodyanoy V., Quan A., Sinnarajah S., Suppiramaniam V., Kessler M., Bahr B. A. (1996): Effects of heparin on the properties of solubilized and reconstituted rat brain AMPA receptors. Neurosci. Lett. 217, 179-183 http://dx.doi.org/10.1016/0304-3940(96)12984-0

Hehl S., Neumcke B. (1993): Negative cooperativity may explain flat concentration-response curves of ATP-sensitive potassium channels. Eur. Biophys. J. 22, 1-4 http://dx.doi.org/10.1007/BF00205806

Hille B. (2001): Ion channels of excitable membranes. Sunderland, MA, Sinauer Associates

Hymel L., Striessnig J., Glossmann H., Schindler H. (1988): Purified skeletal muscle 1,4-dihydropyridine receptor forms phosphorylation-dependent oligomeric calcium channels in planar bilayers. Proc. Natl. Acad. Sci. USA 85, 4290-4294 http://dx.doi.org/10.1073/pnas.85.12.4290 
Imredy J. P., Yue D. T. (1992): Submicroscopic Ca2+ diffusion mediates inhibitory coupling between individual $\mathrm{Ca} 2+$ channels. Neuron 9, 197-207 http://dx.doi.org/10.1016/0896-6273(92)90159-B

Iwasa K., Ehrenstein G., Moran N., Jia M. (1986): Evidence for interactions between batrachotoxin-modified channels in hybrid neuroblastoma-cells. Biophys. J. 50, 531-537 http://dx.doi.org/10.1016/S0006-3495(86)83491-9

Jacobson G. A., Diba K., Yaron-Jakoubovitch A., Oz Y., Koch C., Segev I., Yarom Y. (2005): Subthreshold voltage noise of rat neocortical pyramidal neurones. J. Physiol. 564, 145-160 http://dx.doi.org/10.1113/jphysiol.2004.080903

Keleshian A. M., Yeo G. F., Edeson R. O., Madsen B. W. (1994) Superposition properties of interacting ion channels. Biophys. J. 67, 634-640 http://dx.doi.org/10.1016/S0006-3495(94)80523-5

Keleshian A. M., Edeson R. O., Liu G. J., Madsen B. W. (2000) Evidence for cooperativity between nicotinic acetylcholine receptors in patch clamp records. Biophys. J. 78, 1-12 http://dx.doi.org/10.1016/S0006-3495(00)76568-4

Kensler R. W., Brink P. R., Dewey M. M. (1979): The septum of the lateral axon of the earthworm: a thin section and freeze-fracture study. J. Neurocytol. 8, 565-590 http://dx.doi.org/10.1007/BF01208510

Kentner D., Sourjik V. (2006): Spatial organization of the bacterial chemotaxis system. Curr. Opin. Microbiol. 9, 619-624 http://dx.doi.org/10.1016/j.mib.2006.10.012

Kenyon J. L., Bauer R. J. (2000): Amplitude histograms can identify positively but not negatively coupled channels. J. Neurosci. Methods 96, 105-111 http://dx.doi.org/10.1016/S0165-0270(99)00189-2

Kole M. H., Hallermann S., Stuart G. J. (2006): Single Ih channels in pyramidal neuron dendrites: properties, distribution, and impact on action potential output. J. Neurosci. 26, $1677-1687$ http://dx.doi.org/10.1523/JNEUROSCI.3664-05.2006

Kretzberg J., Egelhaaf M., Warzecha A. K. (2001): Membrane potential fluctuations determine the precision of spike timing and synchronous activity: a model study. J. Comput. Neurosci. 10, 79-97 http://dx.doi.org/10.1023/A:1008972111122

Kung C. (2005): A possible unifying principle for mechanosensation. Nature 436, 647-654 http://dx.doi.org/10.1038/nature03896

Laver D. R., O`Neill E. R., Lamb G. D. (2004): Luminal Ca2+-regulated $\mathrm{Mg} 2+$ inhibition of skeletal RyRs reconstituted as isolated channels or coupled clusters. J. Gen. Physiol. 124, 741-758 http://dx.doi.org/10.1085/jgp.200409092

Liu Y., Dilger J. P. (1993): Application of the one-dimensional and two-dimensional Ising models to studies of cooperativity between ion channels. Biophys. J. 64, 26-35 http://dx.doi.org/10.1016/S0006-3495(93)81337-7

Lundbaek J. A., Birn P., Hansen A. J., Sogaard R., Nielsen C., Girshman J., Bruno M. J., Tape S. E., Egebjerg J., Greathouse D. V., Mattice G. L., Koeppe R. E., 2nd, Andersen O. S. (2004): Regulation of sodium channel function by bilayer elasticity: the importance of hydrophobic coupling. Effects of micelle-forming amphiphiles and cholesterol. J. Gen. Physiol. 123, 599-621 http://dx.doi.org/10.1085/jgp.200308996

Lyford G. L., Strege P. R., Shepard A., Ou Y., Ermilov L., Miller S. M., Gibbons S. J., Rae J. L., Szurszewski J. H., Farrugia G. (2002): Alpha(1C) (Ca(V)1.2) L-type calcium channel mediates mechanosensitive calcium regulation. Am. J. Physiol. Cell Physiol. 283, C1001-1008 http://dx.doi.org/10.1152/ajpcell.00140.2002

Malenka R. C. (2003a): The long-term potential of LTP. Nat Rev Neurosci 4, 923-926 http://dx.doi.org/10.1038/nrn1258

Malenka R. C. (2003b): Synaptic plasticity and AMPA receptor trafficking. Ann. NY Acad. Sci. 1003, 1-11 http://dx.doi.org/10.1196/annals.1300.001

Manivannan K., Ramanan S. V., Mathias R. T., Brink P. R. (1992): Multichannel recordings from membranes which contain gap junctions. Biophys. J. 61, 216-227 http://dx.doi.org/10.1016/S0006-3495(92)81828-3

Martinac B., Adler J., Kung C. (1990): Mechanosensitive ion channels of E. coli activated by amphipaths. Nature 348, 261-263 http://dx.doi.org/10.1038/348261a0

Martinac B. (2004): Mechanosensitive ion channels: molecules of mechanotransduction. J. Cell. Sci. 117, 2449-2460 http://dx.doi.org/10.1242/jcs.01232

Marx S. O., Ondrias K., Marks A. R. (1998): Coupled gating between individual skeletal muscle $\mathrm{Ca} 2+$ release channels (ryanodine receptors). Science 281, 818-821 http://dx.doi.org/10.1126/science.281.5378.818

Marx S. O., Gaburjakova J., Gaburjakova M., Henrikson C., Ondrias K., Marks A. R. (2001): Coupled gating between cardiac calcium release channels (ryanodine receptors). Circ. Res. 88, $1151-1158$ http://dx.doi.org/10.1161/hh1101.091268

Matveev V., Bertram R., Sherman A. (2009): Ca2+ current versus Ca2+ channel cooperativity of exocytosis. J. Neurosci. 29, 12196-12209 http://dx.doi.org/10.1523/JNEUROSCI.0263-09.2009

McCall E., Li L., Satoh H., Shannon T. R., Blatter L. A., Bers D. M. (1996): Effects of FK-506 on contraction and Ca2+ transients in rat cardiac myocytes. Circ. Res. 79, 1110-1121 http://dx.doi.org/10.1161/01.RES.79.6.1110

McCormick D. A., Shu Y., Yu Y. (2007): Neurophysiology: Hodgkin and Huxley model--still standing? Nature 445, E1-2; discussion E2-3

Molina M. L., Barrera F. N., Fernandez A. M., Poveda J. A., Renart M. L., Encinar J. A., Riquelme G., Gonzalez-Ros J. M. (2006): Clustering and coupled gating modulate the activity in KcsA, a potassium channel model. J. Biol. Chem. 281, 18837-18848 http://dx.doi.org/10.1074/jbc.M600342200

Naundorf B., Wolf F., Volgushev M. (2006): Unique features of action potential initiation in cortical neurons. Nature 440, $1060-1063$ http://dx.doi.org/10.1038/nature04610

Navedo M. F., Cheng E. P., Yuan C., Votaw S., Molkentin J. D., Scott J. D., Santana L. F. (2010): Increased coupled gating of L-type $\mathrm{Ca} 2+$ channels during hypertension and Timothy syndrome. Circ. Res. 106, 748-756 http://dx.doi.org/10.1161/CIRCRESAHA.109.213363 
Nedelman J., Wallenius T. (1986): Bernoulli trials, Poisson trials, surprising variances, and Jensen inequality. American Statistician 40, 286-289

Neumcke B., Stampfli R. (1983): Alteration of the conductance of $\mathrm{Na}+$ channels in the nodal membrane of frog nerve by holding potential and tetrodotoxin. Biochim. Biophys. Acta 727, 177-184 http://dx.doi.org/10.1016/0005-2736(83)90382-6

Paoletti P., Ascher P. (1994): Mechanosensitivity of NMDA receptors in cultured mouse central neurons. Neuron 13, 645-655 http://dx.doi.org/10.1016/0896-6273(94)90032-9

Parkinson J. S., Ames P., Studdert C. A. (2005): Collaborative signaling by bacterial chemoreceptors. Curr. Opin. Microbiol. 8, $116-121$ http://dx.doi.org/10.1016/j.mib.2005.02.008

Perozo E., Kloda A., Cortes D. M., Martinac B. (2002): Physical principles underlying the transduction of bilayer deformation forces during mechanosensitive channel gating. Nat. Struct. Biol. 9, 696-703 http://dx.doi.org/10.1038/nsb827

Pleumsamran A., Kim D. (1995): Membrane stretch augments the cardiac muscarinic K+ channel activity. J. Membr. Biol. 148, 287-297 http://dx.doi.org/10.1007/BF00235046

Qin F., Auerbach A., Sachs F. (2000): A direct optimization approach to hidden Markov modeling for single channel kinetics. Biophys. J. 79, 1915-1927 http://dx.doi.org/10.1016/S0006-3495(00)76441-1

Raghuram V., Mak D. O., Foskett J. K. (2001): Regulation of cystic fibrosis transmembrane conductance regulator single-channel gating by bivalent PDZ-domain-mediated interaction. Proc. Natl. Acad. Sci. USA 98, 1300-1305 http://dx.doi.org/10.1073/pnas.98.3.1300

Saito A., Inui M., Radermacher M., Frank J., Fleischer S. (1988): Ultrastructure of the calcium release channel of sarcoplasmic reticulum. J. Cell. Biol. 107, 211-219 http://dx.doi.org/10.1083/jcb.107.1.211

Sakmann B., Neher E. (1995): Single-channel recording. New York, NY, Plenum Press

Schindler H., Spillecke F., Neumann E. (1984): Different channel properties of Torpedo acetylcholine receptor monomers and dimers reconstituted in planar membranes. Proc. Natl. Acad. Sci. USA 81, 6222-6226 http://dx.doi.org/10.1073/pnas.81.19.6222

Schneidman E., Freedman B., Segev I. (1998): Ion channel stochasticity may be critical in determining the reliability and precision of spike timing. Neural. Comput. 10, 1679-1703 http://dx.doi.org/10.1162/089976698300017089

Shahrezaei V., Cao A., Delaney K. R. (2006): Ca2+ from one or two channels controls fusion of a single vesicle at the frog neuromuscular junction. J. Neurosci. 26, 13240-13249 http://dx.doi.org/10.1523/JNEUROSCI.1418-06.2006

Siemens J., Lillo C., Dumont R. A., Reynolds A., Williams D. S., Gillespie P. G., Muller U. (2004): Cadherin 23 is a component of the tip link in hair-cell stereocilia. Nature 428, 950-955 http://dx.doi.org/10.1038/nature02483

Sollner C., Rauch G. J., Siemens J., Geisler R., Schuster S. C., Muller U., Nicolson T. (2004): Mutations in cadherin 23 affect tip links in zebrafish sensory hair cells. Nature 428, 955-959

http://dx.doi.org/10.1038/nature02484

Sourjik V. (2004): Receptor clustering and signal processing in E. coli chemotaxis. Trends Microbiol. 12, 569-576

http://dx.doi.org/10.1016/j.tim.2004.10.003

Sourjik V., Berg H. C. (2002): Binding of the Escherichia coli response regulator $\mathrm{CheY}$ to its target measured in vivo by fluorescence resonance energy transfer. Proc. Natl. Acad. Sci. USA 99, 12669-12674

http://dx.doi.org/10.1073/pnas.192463199

Sourjik V., Berg H. C. (2004): Functional interactions between receptors in bacterial chemotaxis. Nature 428, 437-441 http://dx.doi.org/10.1038/nature02406

Sukharev S., Corey D. P. (2004): Mechanosensitive channels: multiplicity of families and gating paradigms. Sci. STKE 2004, re4

Suppiramaniam V., Vaithianathan T., Manivannan K., Dhanasekaran M., Parameshwaran K., Bahr B. A. (2006): Modulatory effects of dextran sulfate and fucoidan on binding and channel properties of AMPA receptors isolated from rat brain. Synapse 60, 456-464 http://dx.doi.org/10.1002/syn.20319

Tang Q. Y., Qi Z., Naruse K., Sokabe M. (2003): Characterization of a functionally expressed stretch-activated BKca channel cloned from chick ventricular myocytes. J. Membr. Biol. 196, 185-200 http://dx.doi.org/10.1007/s00232-003-0637-8

Tateishi Y., Hattori M., Nakayama T., Iwai M., Bannai H., Nakamura T., Michikawa T., Inoue T., Mikoshiba K. (2005): Cluster formation of inositol 1,4,5-trisphosphate receptor requires its transition to open state. J. Biol. Chem. 280, $6816-6822$ http://dx.doi.org/10.1074/jbc.M405469200

Traynelis S. F., Jaramillo F. (1998): Getting the most out of noise in the central nervous system. Trends Neurosci. 21, 137-145 http://dx.doi.org/10.1016/S0166-2236(98)01238-7

Ursell T., Huang K. C., Peterson E., Phillips R. (2007): Cooperative gating and spatial organization of membrane proteins through elastic interactions. PLoS Comput. Biol. 3, e81 http://dx.doi.org/10.1371/journal.pcbi.0030081

Vaithianathan T., Manivannan K., Kleene R., Bahr B. A., Dey M. P., Dityatev A., Suppiramaniam V. (2005): Single channel recordings from synaptosomal AMPA receptors. Cell. Biochem. Biophys. 42, 75-85 http://dx.doi.org/10.1385/CBB:42:1:075

van Rossum M. C., O’Brien B. J., Smith R. G. (2003): Effects of noise on the spike timing precision of retinal ganglion cells. J. Neurophysiol. 89, 2406-2419 http://dx.doi.org/10.1152/jn.01106.2002

Van Wagoner D. R. (1993): Mechanosensitive gating of atrial ATPsensitive potassium channels. Circ. Res. 72, 973-983 http://dx.doi.org/10.1161/01.RES.72.5.973

Veenstra R. D., Wang H. Z., Beyer E. C., Brink P. R. (1994): Selective dye and ionic permeability of gap junction channels formed by connexin45. Circ. Res. 75, 483-490 http://dx.doi.org/10.1161/01.RES.75.3.483

Wang S., Yue H., Derin R. B., Guggino W. B., Li M. (2000): Accessory protein facilitated CFTR-CFTR interaction, a molecular 
mechanism to potentiate the chloride channel activity. Cell 103, 169-179 http://dx.doi.org/10.1016/S0092-8674(00)00096-9

White J. A., Klink R., Alonso A., Kay A. R. (1998): Noise from voltage-gated ion channels may influence neuronal dynamics in the entorhinal cortex. J. Neurophysiol. 80, 262-269

Xiao R. P., Valdivia H. H., Bogdanov K., Valdivia C., Lakatta E. G., Cheng H. (1997): The immunophilin FK506-binding protein modulates $\mathrm{Ca} 2+$ release channel closure in rat heart. J. Physiol 500, 343-354
Yeo G. F., Edeson R. O., Milne R. K., Madsen B. W. (1989): Superposition properties of independent ion channels. Proc. R. Soc. Lond. B Biol. Sci. 238, 155-170

http://dx.doi.org/10.1098/rspb.1989.0073

Yeramian E., Trautmann A., Claverie P. (1986): Acetylcholine receptors are not functionally independent. Biophys. J. 50, 253-263 http://dx.doi.org/10.1016/S0006-3495(86)83459-2

Received: August 26, 2013

Final version accepted: September 25, 2013 